\title{
Colloidal Silver Nanoparticles Generated in the Presence of Protective Cationic Polyelectrolytes
}

\author{
Andrea B. R. MAYER, ${ }^{\dagger}$ Sven H. HAUSNER, ${ }^{\dagger \dagger}$ and James E. MARK* \\ Department of Chemistry, The University of Cincinnati. \\ Cincinnati, OH $45221-0172$, U.S.A. \\ * Department of Chemistry and the Polymer Research Center, \\ The University of Cincinnati, Cincinnati, OH 45221-0172, U.S.A.
}

(Received February 8, 1999)

\begin{abstract}
Several cationic polyelectrolytes were investigated for their ability to stabilize silver colloids, which were prepared from a silver nitrate $\mathrm{AgNO}_{3}$ precursor by in situ reduction with potassium borohydride $\mathrm{KBH}_{4}$. UV-Vis spectroscopy was employed to characterize the colloidally stable silver samples, and the particle sizes, size distributions, and shapes were determined by transmission electron microscopy. The interactions provided by the silver precursors and colloids with the polymeric matrices were found to have an influence on the nanoparticle features, their optical properties, and the long-term colloidal stability of the dispersions.

KEY WORDS Silver Nanoparticles / Colloid / Cationic Polyelectrolyte /
\end{abstract}

Interest in nanosized colloidal metal nanoparticles protected by polymers is increasing, since these materials can be highly useful for a variety of technological applications. In particular, the size effects and related novel properties of the metal species can be combined with the properties stemming from the polymeric matrix. In this way, materials can be tailored for applications involving optical, catalytic, magnetic, or electronic properties. ${ }^{1-21}$

Silver particles in the nanometer size-range and protected by a polymeric matrix are of interest since they can be useful in many fields. The optical properties provided by such nanosized silver particles are especially intriguing. Depending on the particle size, shape, and agglomeration behavior, silver colloids can show a variety of different colors ranging from the typical yellow to orange, brown and violet. Interestingly, only small amounts of silver are necessary to cause significant optical changes in a composite material. Furthermore, silver nanoparticles are promising candidates for various catalytic applications, especially for electron-transfer and storage or oxidation catalysis. ${ }^{22-27}$

The protective polymer has to be carefully selected, since it can provide a variety of additional functions. A large number of preparative methods for these metalpolymer composite materials involve the presence of the polymer during the reduction from the metal precursor. In this way, the polymer can profoundly influence the particle sizes and morphologies of the resulting metal colloids. A very crucial factor is the influence of the polymer on the materials properties and performance. For instance, the protective polymer surrounding the nanometal and exhibiting its special properties can modify the catalytic and optical properties of the entire metal-polymer system. It is therefore necessary to investigate a broad variety of protective polymers for their effect on such metal colloids.

Water-soluble homopolymers and random copolymers possessing a hydrophobic backbone and hydrophilic side groups are frequently used as stabilizing matrices for metal colloids. ${ }^{1,3}$ For these flexible polymers the stabilization of the metal colloid is based on steric effects. $^{1,28}$

Another interesting class consists of the polyelectrolytes, which are capable of combining both steric and electrostatic stabilization resulting in electrosteric stabilization. This is important for the long-term stabilization of the metal colloids. They are also interesting for a variety of technological applications, such as catalysis, since they can create an electrostatic environment surrounding the metal nanoparticles. This could have an important effect especially if charged or polar reactants are involved. Polyelectrolytes can further provide strong interactions with various metal precursor ions by ion pair formation. Such interactions of the metal precursor with the polymeric matrix turned out to be advantageous for obtaining small particle sizes with a narrow size distribution. ${ }^{8,20}$ For instance, in our previous investigations the use of various cationic polyelectrolytes resulted in small and well-stabilized colloidal gold, palladium, and platinum nanoparticles. ${ }^{18,20}$ In these cases the precursor species were anionic complexes, such as $\left[\mathrm{AuCl}_{4}\right]^{-}$and $\left[\mathrm{PtCl}_{6}\right]^{2-}$. For such anionic precursors the use of anionic polyelectrolytes or partially deprotonated polyacids turned out to be less stabilizing, and the nanoparticles tended to exhibit broader size distributions or agglomerated particles. This is due to the electrostatic repulsion between the anionic polymer side groups and the anionic precursor species, and the fact that anionic counterions are adsorbed on

\footnotetext{
$\uparrow$ Correspondence should be addressed to Department of Chemical Engineering, Chemistry, and Materials Science, Polytechnic University Brooklyn, Six Metro Tech Center, Brooklyn, N.Y. 11201, U.S.A.).

${ }^{+1}$ S.H.H. is currently a graduate student in the group of Prof. Hans Zimmer, Department of Chemistry, The University of Cincinnati, Cincinnati, OH 45221-0172, U.S.A.
} 


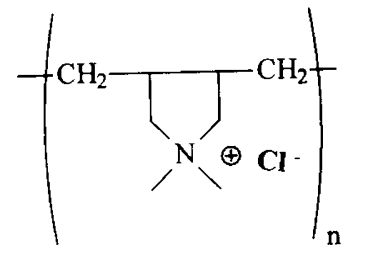

Poly(diallyldimethyl ammonium chloride)

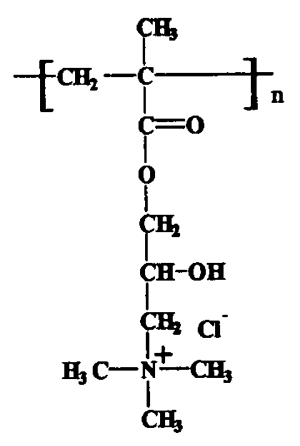

Poly(2-hydroxy-3-methacryloxypropyltrimethyl ammonium chloride)

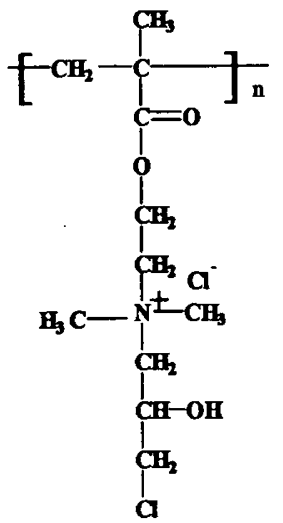

Poly(3-chloro-2-hydroxypropyl-2methacryl-oryethyldimethyl ammonium chloride)

Figure 1. Structures of the cationic polyelectrolytes.

the resulting colloid surfaces.

In the present investigations several cationic polyelectrolytes incorporating quaternary ammonium groups were investigated for their ability to stabilize colloidal silver nanoparticles, and their effect on the nanoparticle features and long-term stabilization. For comparison, the nonionic poly(dimethylamine- $c o$-epichlorohydrine) was included. The silver colloids were prepared from a silver nitrate $\mathrm{AgNO}_{3}$ precursor by the in situ reduction with potassium borohydride $\mathrm{KBH}_{4}$ in aqueous medium. In contrast to the anionic precursor species investigated earlier, ${ }^{18,20}$ in this case the precursor species is cationic, namely the $\mathrm{Ag}^{+}$aquo-complex. This should result in differences with respect to the nanoparticle features and the colloidal stability of the samples for the various polymeric matrices involved. The particle sizes, shapes, and size distributions of the colloidal dispersions were investigated by transmission electron microscopy (TEM). UV-Vis spectroscopy was employed for additional characterization of the optical properties of the colloids. The type of the protective polymer can have a large influence on the nanoparticle features, the optical properties, and the colloidal stability of the samples.

\section{EXPERIMENTAL}

\section{Chemicals and Reagents}

Silver nitrate $\left(\mathrm{AgNO}_{3}\right)$ and potassium borohydride $\left(\mathrm{KBH}_{4}\right)$ were obtained from Aldrich. Poly(dimethylamine-co-epichlorohydrin) (molecular weight, $M_{w}$, not available from supplier) and the poly(diallyldimethyl ammonium chlorides) were purchased from Aldrich. The molecular weights of the poly(diallyldimethyl ammonium chlorides) were given by the supplier as follows: average $M_{w} \sim 200000-350000$ (medium $M_{w}$ ) and average $M_{w} \sim 400000-500000$ (high $M_{w}$ ). Poly(2-hydroxy-3methacryloxypropyltrimethyl ammonium chloride) $\left(M_{w}\right.$ not available from supplier) and poly(3-chloro-2-hydroxypropyl-2-methacryloxyethyldimethyl ammonium chloride) $\left(M_{w} \sim 50000\right)$ were obtained from Polysciences. The structures of the cationic polyelectrolytes are depicted in Figure 1.

\section{Colloid Preparation}

All glassware employed was cleaned with concentrated nitric acid before use. An aqueous solution of an excess of $\mathrm{KBH}_{4}$ was prepared just before use and rapidly added to the stirred aqueous solutions containing $\mathrm{AgNO}_{3}$ $\left(6.8 \times 10^{-4} \mathrm{M}\right)$ and the nonionic polymer or cationic polyelectrolytes in a mass ratio of polymer: silver = $25: 1$. UV-Vis spectra were taken immediately after the reduction, and further UV-Vis spectra were taken after $2 \mathrm{~h}$ storage in air and ambient light. Additional spectra were recorded after storage in air and in the dark for several months to explore the colloidal long-term stability of the samples.

\section{Characterization}

Transmission electron micrographs were taken with a JEOL-100 CX II transmission electron microscope (operating at an acceleration voltage of $80 \mathrm{kV}$ ) in order to obtain the sizes, shapes, and size distributions of the silver nanoparticles. The samples were prepared by placing a drop of the colloidal dispersion on a carbon/ formvar-coated copper grid (placed onto a filter paper to remove excess solvent) and letting the solvent evaporate at room temperature. Further TEM samples were prepared for two examples [silver colloids in the presence of poly(diallyldimethyl ammonium chloride) and poly(2-hydroxy-3-methacryloxypropyltrimethyl ammonium chloride)] after storage of the colloidal dispersions for 7-8 months in the dark, and the micrographs of these stored samples were taken with a Phillips EM 400 transmission electron microscope (operated at $80 \mathrm{kV}$ ). The particle sizes were measured with a comparator and the number average particle sizes and size distributions were determined based on the measurement of at least 150 particles. UV-Vis spectra were recorded with a Milton Roy Spectronic 3000 Array instrument (using $10 \mathrm{~mm}$ pathlength quartz cuvettes) from 250 to $600 \mathrm{~nm}$.

\section{RESULTS}

\section{Freshly-Prepared Samples}

The results obtained by TEM, that is, the number- 
Table I. Transmission electron microscopy results and UV-Vis spectroscopic data for silver colloids reduced by $\mathrm{KBH}_{4}$

\begin{tabular}{|c|c|c|c|c|c|}
\hline Polymer & $\begin{array}{l}\text { Average particle } \\
\text { size/nm (std. dev.) }\end{array}$ & Particle features & Color & $\lambda_{\max } / \mathrm{nm}$ & FWHM \\
\hline \multicolumn{6}{|l|}{ Nonionic polymer: } \\
\hline $\begin{array}{l}\text { Poly(dimethylamine-co-epichloro- } \\
\text { hydrin) } 50: 50(\mathrm{~mol} .)\end{array}$ & $20.4(6.7)$ & $\begin{array}{l}\text { Spherical, } \\
\text { several "pairs" }\end{array}$ & Yellow & 417.8 & 76 \\
\hline \multicolumn{6}{|l|}{ Cationic polyelectrolytes: } \\
\hline $\begin{array}{l}\text { Poly(diallyldimethyl ammonium } \\
\text { chloride), high mol. wt. }\end{array}$ & $28.3(12.1)$ & a & $\begin{array}{l}\text { Deep yellow- } \\
\text { orange }\end{array}$ & $\begin{array}{l}401.4 \\
398.1^{\mathrm{d}}\end{array}$ & $\begin{array}{l}60 \\
49^{d}\end{array}$ \\
\hline $\begin{array}{l}\text { Poly(diallyldimethyl ammonium } \\
\text { chloride), medium mol. wt. }\end{array}$ & $\sim 100$ & a & $\begin{array}{l}\text { Deep yellow- } \\
\text { orange }\end{array}$ & $\begin{array}{l}402.4 \\
396.0^{\mathrm{e}}\end{array}$ & $\begin{array}{l}79 \\
40^{e}\end{array}$ \\
\hline $\begin{array}{l}\text { Poly(2-hydroxy-3-methacryloxy- } \\
\text { propyltrimethyl ammonium } \\
\text { chloride) }\end{array}$ & $38.6(26.0)$ & a & Yellow-orange & $\begin{array}{l}404.2 \\
399.2^{\mathrm{e}}\end{array}$ & $\begin{array}{l}77 \\
62^{\mathrm{e}}\end{array}$ \\
\hline $\begin{array}{l}\text { Poly(3-chloro-2-hydroxypropyl-2- } \\
\text { methacryloxyethyldimethyl } \\
\text { ammonium chloride) }\end{array}$ & $13.9(19.5)$ & a & Yellow-orange & $\begin{array}{l}422 \\
417.5^{\mathrm{e}}\end{array}$ & $\begin{array}{l}90 \\
83^{\mathrm{e}}\end{array}$ \\
\hline $\begin{array}{l}\text { After storage for } 7-8 \text { months: } \\
\text { Poly(diallyldimethyl ammonium } \\
\text { chloride), high mol. wt. }\end{array}$ & $\begin{array}{l}2.3(1.7) \\
1.6(0.7)^{\mathrm{c}}\end{array}$ & $\mathrm{b}$ & Yellow & \multicolumn{2}{|c|}{ After storage for $7-8$ months: } \\
\hline $\begin{array}{l}\text { Poly(2-hydroxy-3-methacryloxy- } \\
\text { propyltrimethyl ammonium } \\
\text { chloride) }\end{array}$ & $\begin{array}{l}1.9(2.3) \\
1.6(0.3)^{\mathrm{c}}\end{array}$ & $\mathrm{b}$ & Yellow & 398.5 & 54 \\
\hline
\end{tabular}

a Spherical and "close-to-spherical" particles in coexistence with nanosized single crystals exhibiting mainly rectangular profiles and fewer triangular profiles. " Many very small spherical particles (of about $1-2 \mathrm{~nm}$ diameter) in coexistence with larger ones (spherical, oval and "close-to-spherical" particles; no single crystals). "Average particle diameters for the particles excluding the coexisting larger ones. ${ }^{\mathrm{d}} \mathrm{UV}-\mathrm{Vis}$ spectrum recorded after $1 \mathrm{~h}$ storage in air and ambient light. ${ }^{\mathrm{e}} \mathrm{UV}$-Vis spectra recorded after $2 \mathrm{~h}$ storage in air and ambient light.

average particle size (and standard deviation) and the particle shapes for the silver colloids protected by poly(dimethylamine-co-epichlorohydrine) and the cationic polyelectrolytes are summarized in Table I. The UV-Vis spectroscopic data, along with the colors of the samples, obtained immediately and $2 \mathrm{~h}$ after the reduction are included as well.

Several cationic polyelectrolytes based on quaternary ammonium groups were investigated, all of them possessing chloride counterions. All cases gave colloidally-stable silver sols, which could be stored for several months. For comparison, one nonionic polymer, namely poly(dimethylamine-co-epichlorohydrin), was investigated as well. This polymer contains amino units, which can be protonated and which can also act as ligands for the silver cation $\mathrm{Ag}^{+} .{ }^{29}$

The poly(dimethylamine-co-epichlorohydrin) copolymer as a protective matrix initially yielded a yellow silver sol which was stable for several days. The average particle size obtained by TEM was about $20 \mathrm{~nm}$, as can also be seen from the TEM micrograph depicted in Figure 2. For this polymer several "paired" particles were found, in coexistence with separate single silver particles. However, after about 2-3 weeks storage in air the formation of a white precipitate and a colorless solution indicated poor long-term colloidal and chemical stabilization of this sample, most probably due to partial reoxidation and complex formation of the silver species with the amino units.

Typical TEM micrographs for the stable samples stabilized by the cationic polyelectrolytes are shown in Figure 3 for silver nanoparticles protected by poly(diallyldimethyl ammonium chloride), and in Figure 4 for silver particles in the presence of poly(2-hydroxy-3methacryloxypropyltrimethyl ammonium chloride). Usually, for these samples a variety of coexisting particular shapes are found, that is, spherical and

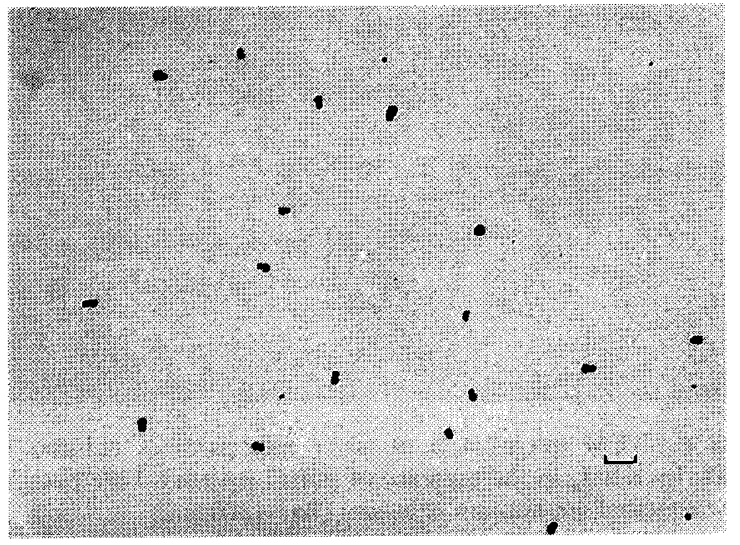

Figure 2. TEM micrograph of silver nanoparticles reduced from $\mathrm{AgNO}_{3}$ by $\mathrm{KBH}_{4}$ in the presence of poly(dimethylamine-coepichlorohydrin) $(\mathrm{Bar}=105 \mathrm{~nm})$.

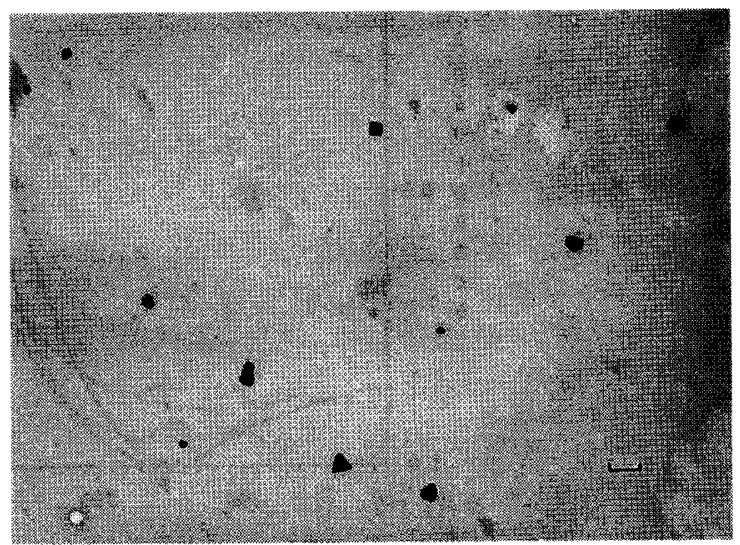

Figure 3. TEM micrograph of silver nanoparticles reduced from $\mathrm{AgNO}_{3}$ by $\mathrm{KBH}_{4}$ in the presence of poly(diallyldimethy! ammonium chloride), medium molecular weight $(B a r=400 \mathrm{~nm})$. 
close-to-spherical, somewhat irregular shapes, in addition to nanosized single crystals. For the nanosized single crystals rectangular profiles are predominant, in addition to some triangular profiles.

Even though somewhat larger particle shapes are initially present, the dispersions were colloidally stable for several months with no precipitation occurring. The UV-Vis spectroscopic data for the samples are also listed in Table I. They were recorded immediately after the reduction, and also after $2 \mathrm{~h}$ storage in air and ambient light.

The UV-Vis spectra taken immediately after the reduction show a variation of the plasmon absorption band for different types of the protective polymers. A peak at about $400-405 \mathrm{~nm}$ and a minimum around $320 \mathrm{~nm}$ were found for the poly(diallyldimethyl ammonium chloride) and poly (2-hydroxy-3-methacryloxypropyltrimethyl ammonium chloride) samples. Such UV-Vis spectra are typically found for nanosized silver colloids. In addition, a peak at about $275 \mathrm{~nm}$ shows the existence of the $\mathrm{Ag}_{4}{ }^{2+}$ species, which is reported to be formed during the two-step reduction process by borohy-

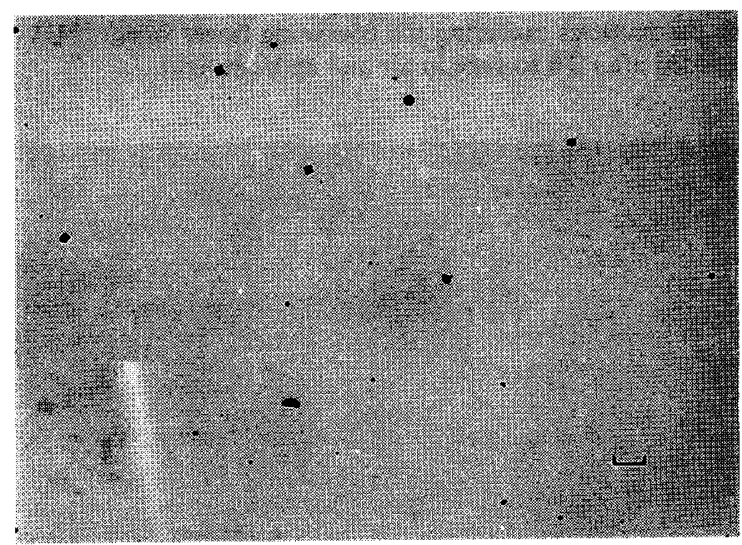

Figure 4. TEM micrograph of silver nanoparticles reduced from $\mathrm{AgNO}_{3}$ by $\mathrm{KBH}_{4}$ in the presence of poly(2-hydroxy-3-methacryloxypropyltrimethyl ammonium chloride) $(\mathrm{Bar}=300 \mathrm{~nm})$. drides. ${ }^{30,31}$ The UV-Vis spectrum for the sample involving poly(diallyldimethyl ammonium chloride) is depicted in Figure 5a.

The sample involving the nonionic poly(dimethylamine-co-epichlorohydrin) shows a red shift, that is plasmon absorption at a higher wavelength. In this case the plasmon absorption peak is located at about $418 \mathrm{~nm}$. Also in this case an additional weak peak around $280 \mathrm{~nm}$ was recorded for the freshly prepared sample, showing the presence of the $\mathrm{Ag}_{4}{ }^{2+}$ species. The largest red shift was found for the poly(3-chloro-2-hydroxypropyl-2methacryloxyethyldimethyl ammonium chloride), with a plasmon absorption band at $422 \mathrm{~nm}$. This sample also shows the largest bandwidth (full width at half maximum, FWHM) of $90 \mathrm{~nm}$ (see UV-Vis spectrum depicted in Figure 5b).

The stable samples involving the cationic polyelectrolytes were investigated further, and UV-Vis spectra were also recorded after two hours storage in air and ambient light. These UV-Vis spectrocopic data are also included in Table I. Upon standing a shift to shorter wavelengths ("blue shift") and a significant narrowing of the absorption plasmon band were observed, as can be seen from the representative UV-Vis spectrum for silver nanoparticles protected by poly(diallyldimethyl ammonium chloride) shown in Figure 5c.

\section{Stored Samples}

The blue shift of the absorption plasmon band seemed to be completed after a few hours, since a less drastic change was found after storage in air and in the dark for $7-8$ months. The UV-Vis spectroscopic data for the samples investigated after this storage time are listed in Table I. An example is shown in Figure $5 \mathrm{~d}$ for silver nanoparticles protected by poly(diallyldimethyl ammonium chloride), recorded after storage of the diluted sample for $7-8$ months. Considering the long storage period, the differences relative to the UV-Vis spectra obtained after two hours are quite small.

TEM micrographs were therefore taken for these two

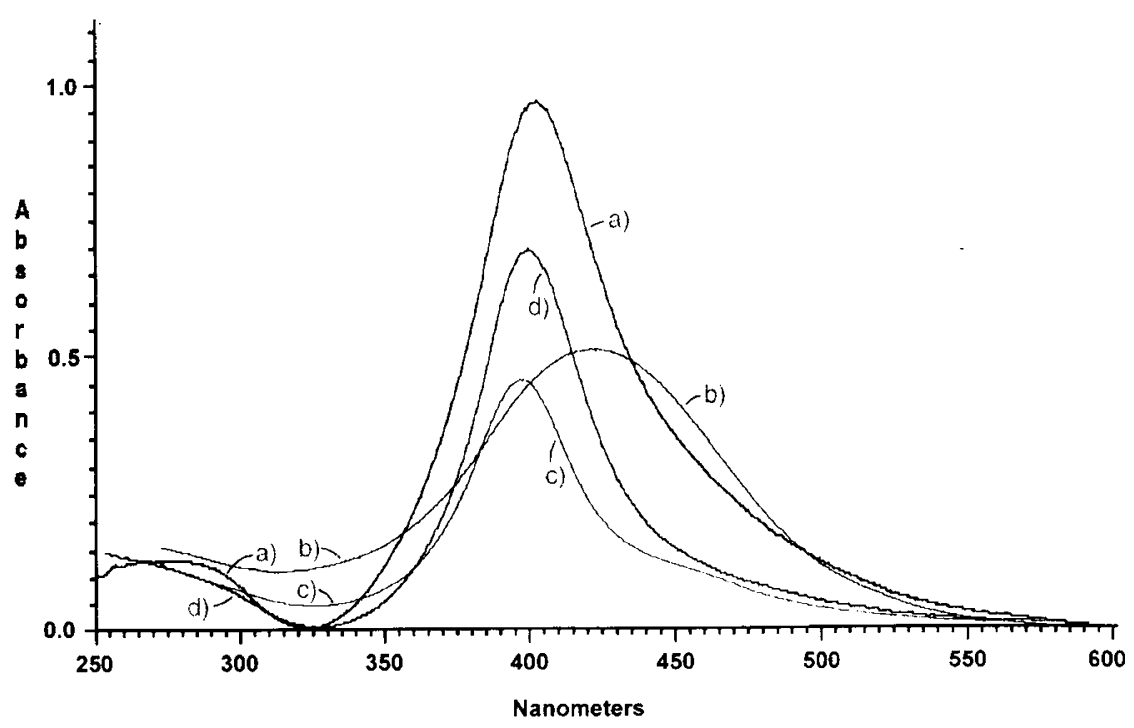

Figure 5. UV-Vis spectra for silver nanoparticles (a) protected by poly(diallyldimethyl ammonium chloride), recorded immediately after the reduction from $\mathrm{AgNO}_{3}$ by $\mathrm{KBH}_{4}$, (b) protected by poly(3-chloro-2-hydroxypropyl-2-methacryloxyethyldimethyl ammonium chloride), recorded immediately after the reduction from $\mathrm{AgNO}_{3}$ by $\mathrm{KBH}_{4}$, (c) protected by poly(diallyldimethyl ammonium chloride), recorded two hours after the reduction from $\mathrm{AgNO}_{3}$ by $\mathrm{KBH}_{4}$, and (d) protected by poly(diallyldimethyl ammonium chloride), recorded after storage for 7--8 months. 


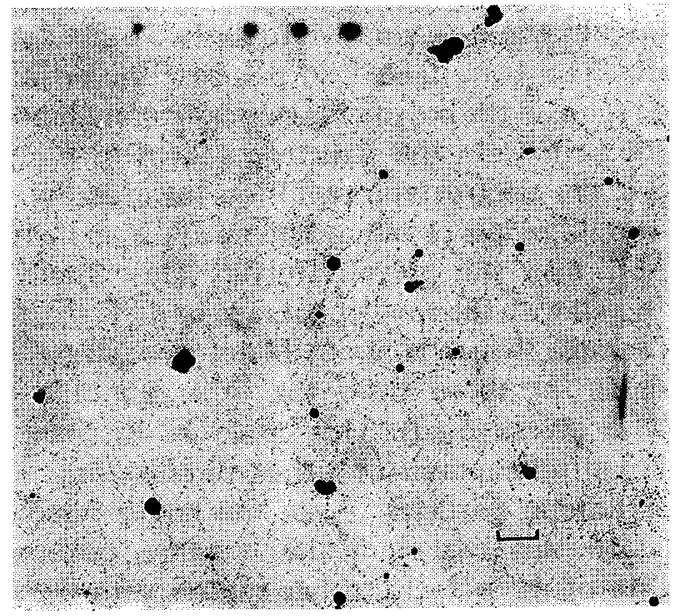

Figure 6. TEM micrograph of silver nanoparticles reduced from $\mathrm{AgNO}_{3}$ by $\mathrm{KBH}_{4}$ in the presence of poly(2-hydroxy-3-methacryloxypropyltrimethyl ammonium chloride), obtained after storage for $7-8$ months $(\operatorname{Bar}=75 \mathrm{~nm})$.

samples after storage and the results given in Table I. In both cases the colloidal dispersions were still yellow and clear. The TEM micrograph in Figure 6 shows the example of silver nanoparticles protected by poly(2hydroxy-3-methacryloxypropyltrimethyl ammonium chloride). The TEM micrographs reveal a significant change of the particle features when compared to those taken immediately after the reduction. Many very small spherical particles (of about $1-2 \mathrm{~nm}$ diameter) are mainly found, in coexistence with fewer larger ones. The average particle size is drastically reduced, and single crystal features are no longer evident. The smallest silver clusters typically form a "network', distributed evenly throughout the sample, as can be seen from the micrograph in Figure 6. Since the colloidal dispersions were still of the typical yellow color after this long storage time, it seems that it is small $\mathrm{Ag}^{\circ}$ clusters of a certain defined size being stabilized by the matrix. These result in the plasmon absorption peak at $398 \mathrm{~nm}$, found for both samples.

\section{DISCUSSION}

\section{Freshly-Prepared Samples}

Several reasons could explain the various particle features found by TEM investigations for the freshlyprepared samples protected by the cationic polyelectrolytes. The different nanoparticle shapes indicate that different growth and agglomeration processes are effective, occurring either simultaneously or in consecutive steps. Also the observed color change during the colloid preparation is an indication for multi-step and secondary growth and agglomeration processes: immediately after the addition of the borohydride solution intense yellow silver sols were obtained which turned a deep orange within the first seconds after the reduction process. Upon standing the silver sols turned yellow again, and remained that color during several months storage.

Usually the formation of nanosized single crystals is associated with a slower, controlled particle growth or "templating effects", whereas the formation of somewhat larger, irregular shapes are often found for faster particle growth and agglomeration processes. It is also likely, that such irregular shapes are caused by a different nucleation species, such as the silver chloride $\mathrm{AgCl}$ or silver oxide $\mathrm{Ag}_{2} \mathrm{O}$, which can also be formed during preparation. It is known that $\mathrm{Ag}^{+}$ions are highly sensitive towards chloride anions, which are present in excess, stemming from the polyelectrolyte counterions. This could result in the formation of colloidal silver chloride $\mathrm{AgCl}$. A large excess of chloride ions, however, could also lead to the formation of a soluble dichloro silver complex $\left[\mathrm{AgCl}_{2}\right]^{-},{ }^{32}$ which could then act as a complex counterion for the cationic polyelectrolytes, forming ion pairs. Furthermore, the formation of silver oxide $\mathrm{Ag}_{2} \mathrm{O}$ in air has been reported in the literature. ${ }^{30,33}$

Thus, a combination of several effects and different types of species forming the initial nuclei could be involved, and causing the observed variations of particle shapes:

Colloidal Semiconductor Nuclei and Particles. As explained above, it is possible, that in several cases the nuclei for particle growth are not $\mathrm{Ag}^{\circ}$ clusters, but small colloidal $\mathrm{AgCl}$ and/or $\mathrm{Ag}_{2} \mathrm{O}$ clusters/particles. These would act either as nucleation sites for the silver nanoparticle growth, or being precursors, and in turn reduced to $\mathrm{Ag}^{\circ}$. Since the colloidal dispersions remained clear during preparation these semiconductor particles are most probably present in the colloidal state. Both the $\mathrm{AgCl}$ and $\mathrm{Ag}_{2} \mathrm{O}$ species can adsorb additional silver ions $\mathrm{Ag}^{+}$on their surface, which then can be reduced to $\mathrm{Ag}^{\circ} \cdot{ }^{30}$ In particular, the irregular and close-to-spherical shapes could be due to colloidal $\mathrm{AgCl}$ and $\mathrm{Ag}_{2} \mathrm{O}$ being reduced to $\mathrm{Ag}^{\circ}$ nanoparticles.

Negatively-Charged Silver Complexes. The sensitivity of the silver species towards chloride, which is present in excess from the polyelectrolyte, could cause the formation of silver chloride. It could also result in the formation of the complex $\left[\mathrm{AgCl}_{2}\right]^{-}$anion, ${ }^{32}$ which can also be formed as a surface complex on the silver nanoparticles. Such negatively-charged species should interact well with the cationic polyelectrolyte. This type of surface complex formation could also be responsible for the changes in the particle features and the stabilization of the system by the cationic polyelectrolytes during longer storage periods.

Positively-Charged Silver Species. The positively charged $\mathrm{Ag}^{+}$precursor ions have a low tendency to interact with the cationic polyelectrolytes, due to electrostatic repulsion. Also, it is reported that the reduction of $\mathrm{AgNO}_{3}$ by borohydrides is a two-step reduction process, involving the initial formation of the $\mathrm{Ag}_{4}{ }^{2+}$ cation. This species shows an absorption peak in the UV-Vis spectrum at about $275 \mathrm{~nm} .{ }^{30,31}$ The initially weaker interaction with the protective cationic polyelectrolyte due to electrostatic repulsion can cause the somewhat larger average particle sizes (mostly in the range of 15 to $40 \mathrm{~nm}$ ) initially observed for the samples, in contrast to the use nonionic protective polymers or polyacids as protective matrices. ${ }^{34}$

Thus it is very likely that different silver species and surface effects are involved in the particle growth and ripening process, and are responsible for the variation of the particle shapes. Therefore it is probable that the 
larger particles seen by TEM are composites of various silver species $\left(\mathrm{Ag}^{+}\right.$and $\left.\mathrm{Ag}^{\circ}\right)$ which can be initially formed, whereas the UV-Vis plasmon absorption stems from nanosized zerovalent silver $\mathrm{Ag}^{\circ}$. Therefore the observed UV-Vis spectra should not be directly correlated to the particle sizes as observed by TEM.

For the nonionic polymer and poly(3-chloro-2hydroxypropyl-2-methacryloxyethyldimethyl ammonium chloride) a red shift was found in comparison to the other cationic polyelectrolytes investigated. Since all reduction conditions and solutions were the same for all samples, and no correlation with the observed particle sizes could be found, the observed shifts of the plasmon bands within the samples can be ascribed to the presence of the different polymers. Generally, a red shift in the UV-Vis spectra can have several reasons: ${ }^{30,33,35-37}$ (i) matrix effects from the polymer, (ii) oxidation processes, and (iii) reduced interactions with nucleophiles (such as chloride ions) or adsorption of positively-charged species.

Since the reductions were already performed in air, red shifts due to partial oxidation by ambient oxygen, as observed by Henglein ${ }^{33}$ for samples prepared in inert atmosphere and then exposed to ambient air, cannot be expected. Since the partial oxidation of silver nanoparticles was found to proceed very rapidly (significant changes have been found by Henglein already after $1.5 \mathrm{~min}$, and further changes came to a halt after about $20 \mathrm{~min})^{33}$ these effects should be already present for the samples investigated here "from the beginning". The differences in the positions of the plasmon peaks for the different samples, and further shifts observed upon storage should stem from the different polymers used and their influence on ion interactions.

The positively-charged precursor and nucleation species $\left(\mathrm{Ag}^{+}, \mathrm{Ag}_{4}{ }^{2+}\right.$, and adsorbed positive silver ions on the formed nanoparticles) can cause both a reduced interaction with the cationic polyelectrolytes (due to electrostatic repulsion) and an increased interaction/ adsorption of nucleophilic species, such as chloride ions. This could explain the absorption at the similar, lower wavelengths for the poly(diallyldimethyl ammonium chloride) and poly (2-hydroxy-3-methacryloxypropyltrimethyl ammonium chloride) samples. For these cationic polyelectrolytes the positive charge along the polymer backbone is only a little hindered, which therefore should result in a lower tendency of these polymers to initially interact with the positively-charged silver species.

In comparison, the poly(dimethylamine-co-epichlorohydrin) sample shows a red shift of more than $10 \mathrm{~nm}$ for the freshly prepared sample. Since in this case no additional excess of nucleophilic species (chloride ions) is present, the effect is likely due to both a matrix effect of the polymer and the increased adsorption of positively-charged silver species on existing nanoparticles. The amino units of the polymer can interact with the silver species by complex formation. This should result in a stronger interaction with the polymer, which could also displace adsorbed anions (e.g., the nitrate counterions from the $\mathrm{AgNO}_{3}$ precursor). ${ }^{38}$ Indeed the change to colorless in the course of storage indicated the reoxidation for this sample.

The largest red shift was found for the poly(3-chloro- 2-hydroxypropyl-2-methacryloxyethyldimethyl ammonium chloride) sample. In contrast to the two other cationic polyelectrolytes investigated, in this case the positivelycharged region in the polymer is sterically more hindered, as can be seen from the structure shown in Figure 1. Therefore, the electrostatic repulsion towards the silver species should be less pronounced and allow an increased interaction with the polyelectrolyte. This would also correlate well with the smaller average particle size found for this sample (which is about $14 \mathrm{~nm}$ in contrast to the $25-100 \mathrm{~nm}$ for those from the other cationic polyelectrolytes investigated). This could be ascribed to a better initial interaction (and thus stabilization) of the silver-containing particles. Also, the larger bandwidth observed in this case indicates a more pronounced interaction of the polyelectrolyte matrix with the silver species. $^{22,36}$ The additional positively-charged field surrounding the silver species could then result in an additional red shift in this case.

After $2 \mathrm{~h}$ storage a blue shift of the plasmon band was observed for the samples involving the cationic polyelectrolytes. Generally such blue shifts can be ascribed to the following effects: ${ }^{22,30,35,36}$ (i) the adsorption of nucleophiles and the associated accumulation of electrons on the silver particles and (ii) a decrease in particle size (which is, however, often associated by a broadening of the bandwidth). ${ }^{36}$

A surface effect stemming from adsorbed nucleophiles is likely, due to the presence of a large quantity of negatively charged counterions, both from the silver precursor (nitrate $\mathrm{NO}_{3}{ }^{-}$) and the cationic polyelectrolytes (chloride $\mathrm{Cl}^{-}$counterions). Also, after completion of the reduction, there is no longer any adsorption of positively-charged silver species (they are completely reduced), and the adsorption of the negatively-charged counterions can become predominant. These counter anions can adsorb on the nanoparticle surface, resulting in a negative surface charge or the formation of surface complexes. A negative surface charge of, for instance, gold and silver colloids due to adsorption of anions has been frequently observed in the literature, ${ }^{38}$ and has been ascribed to "super equivalent" specific adsorption, overcompensating the positive charge on the metal particles. Also, Henglein has reported that anions which form complexes or insoluble salts with silver ions are especially strongly adsorbed on silver particles. ${ }^{39}$ This negative surface charge may also be responsible for the stabilization of the colloids by the cationic polyelectrolytes, by providing improved interaction with the positively charged polyelectrolyte. Even after storage for $7-8$ months the dispersions are of a yellow color with no trace of precipitation. Figure 7 shows a schematic illustration of a particle with adsorbed layers of positive and negative ions, and subsequent interaction with positively-charged components of a polyelectrolyte.

According to the literature, such observed blue shifts can also be ascribed to surface effects stemming from adsorbed silver clusters on the particles. Thus, an initial red shift can occur with the withdrawal of electron density from adsorbed (positively-charged) silver ions. The transfer of electron density from adsorbed silver metal particles to colloidal silver oxide (which can 


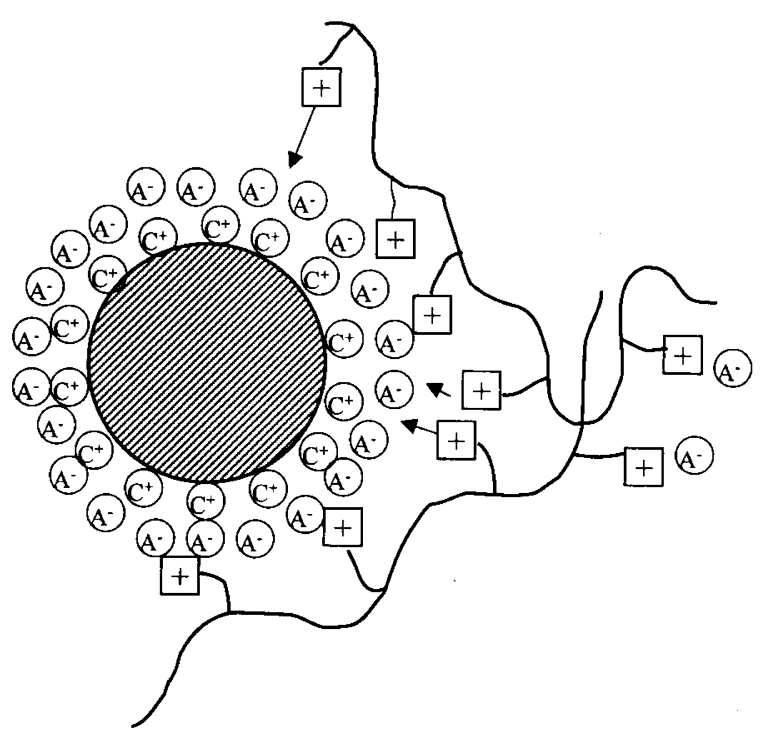

Figure 7. Schematic illustration of a nanoparticle with adsorbed layers of positive and negative ions, resulting in a negative surface charge due to "overcompensation", and subsequent interaction with cationic components of the polyelectrolytes, due to electrostatic attraction.

occur during the formation of small silver particles on the surface of $\mathrm{Ag}_{2} \mathrm{O}$ ), or the dissolution of the silver oxide support during an autocatalytic redox process, can then subsequently cause a blue shift during reaction. ${ }^{30}$

\section{Stored Samples}

As mentioned, the observed blue shift during storage could furthermore be associated with a decrease of the particle size of the samples during aging. It should be noted that after $7-8$ months storage as diluted dispersions for the two samples involving the cationic poly(diallyldimethyl ammonium chloride) and poly(2hydroxy-3-methacryloxypropyl-trimethyl ammonium chloride), the plasmon absorption band located at the same wavelength, $398 \mathrm{~nm}$ was found. Figure $5 \mathrm{~d}$ shows the respective UV-Vis spectrum for the poly(diallyldimethyl ammonium chloride) sample after long-term storage. This wavelength is typical for very small silver particles, ${ }^{30,33,40,41}$ and could point to the same silver species finally stabilized by the polyelectrolytes.

Indeed the measurement of merely the small clusters excluding the coexisting larger ones (which could involve $\mathrm{AgCl}$ or $\mathrm{Ag}_{2} \mathrm{O}$ ) give an average particle size of about $1.6 \mathrm{~nm}$ for both the poly(diallyldimethyl ammonium chloride) and the poly(2-hydroxy-3-methacryloxypropyltrimethyl ammonium chloride) samples. An enlarged TEM micrograph showing a section with the small spherical nanoparticles is presented in Figure 8 for the poly(diallyldimethyl ammonium chloride) sample. This particle size stemming from the most frequent particle size and surrounded by the same type of polymer matrix (both are cationic polyelectrolytes with chloride counter anions and less sterically-hindered positive charges) could be responsible for the identical plasmon absorptions found after this long storage time.

These results differ from observations made for gold nanoparticles protected by cationic polyelectrolytes and

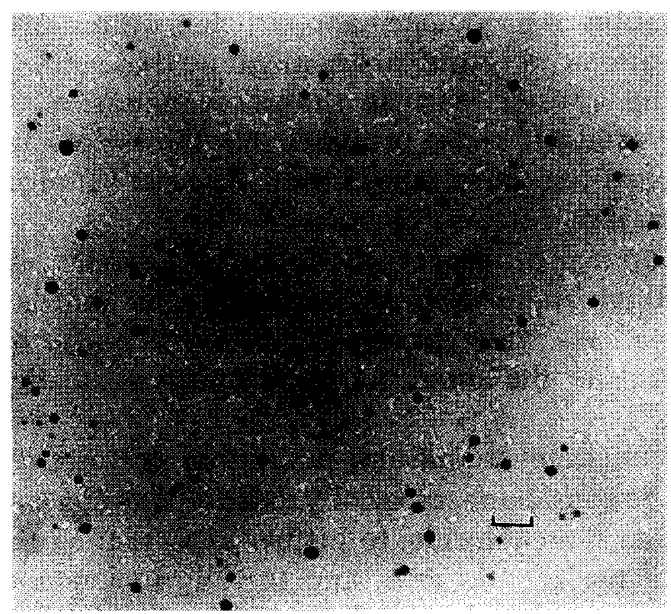

Figure 8. TEM micrograph of silver nanoparticles reduced from $\mathrm{AgNO}_{3}$ by $\mathrm{KBH}_{4}$ in the presence of poly(diallyldimethyl ammonium chloride), high molecular weight, obtained after storage for $7-8$ months $($ Bar $=35 \mathrm{~nm})$.

stored under similar conditions for several months. ${ }^{18}$ In these cases not only the UV-Vis spectra changed very little, but also the TEM pictures revealed only slight increases of the average particle diameters (which could be expected from "regular" aging effects and Ostwald ripening during such long storage times of several months). However, in these cases the initial formation of nanocomposites involving gold ions was not as favored, and the resulting gold nanoparticles are also less subject to dissolution due to complex formation. Thus the long-term storage effects of cationic polyelectrolytes seem to depend strongly on various factors, such as the metal type and its associated chemistry, and the preparation conditions.

Several effects could contribute to the observed drastic decrease of particle sizes observed here for the silver samples. Obviously some "dissolution" processes took place. The findings ressemble observations made by Nedeljkovic and Patel for $\mathrm{AgBr} / \mathrm{Ag}$ nanocomposite particles. ${ }^{42}$ In their investigations the TEM micrographs showed both larger and smaller irregular particles, and the larger particles have been assigned to $\mathrm{AgBr} / \mathrm{Ag}$ nanocomposites. Upon addition of ammonia, which can dissolve silver bromide by complex formation, only small silver particles possessing diameters of $5 \mathrm{~nm}$ or less have been found by TEM. The color of these sols showed the characteristic yellow for silver nanoparticles. Thus, the larger particles, being composites incorporating $\mathrm{AgCl}$ and $\mathrm{Ag}_{2} \mathrm{O}$ could be dissolved by complex formation involving either the excess of chloride counterions or the ammonium groups (both stemming from the polyelectrolyte). This could mean that during the long-term storage certain cluster sizes are stabilized by the cationic polyelectrolytes.

\section{CONCLUSIONS}

Cationic polyelectrolytes are interesting candidates for the stabilization of colloidal silver nanoparticles. Due to the interplay of positive and negative charges, a variety of nanoparticle shapes can be obtained. Further optimization could result in the exclusive formation and 
stabilization of particular nanoparticle features, and the use of cationic polyelectrolytes might be thus suitable for obtaining unusual particle shapes or nanosized silver single crystals. It is also probable that the use of cationic polyelectrolytes can result in the long-term stabilization of small, defined silver clusters.

The optical properties of the metal-polymer systems are strongly influenced by the polymeric matrix and surface effects. Such surface effects may be also highly important for the long-term stabilization of small silver clusters.

For future investigations, additional polyelectrolytes should be considered, especially those containing counter ions other than chloride, to further explore the role and effect of the counter ions, and to fully understand the interplay of positive and negative charges during nanoparticle generation, growth, and stabilization.

Acknowledgments. A.M. would like to thank Professor Hans Zimmer, Department of Chemistry, The University of Cincinnati for generously providing his laboratory space and facilities, and Professor Randal E. Morris, Department of Cell Biology, Neurobiology, and Anatomy, The University of Cincinnati Medical Center, for the training he provided in his electron microscopy facility. A. M. is also indebted to Professor Adi Eisenberg, Department of Chemistry, McGill University, for providing access to a transmission electron microscope and providing laboratory space during manuscript preparation. Access to the UV-Vis instrument was provided by Professor R. Marshall Wilson, Department of Chemistry, The University of Cincinnati. Financial support was provided through the National Science Foundation Grant DMR-9422223 (Polymers Program, Division of Materials Research).

\section{REFERENCES}

1. J. S. Bradley, in "Clusters and Colloids. From Theory to Applications," G. Schmid, Ed., VCH, Weinheim, 1994.

2. U. Kreibig and M. Vollmer, in "Optical Properties of Metal Clusters," Springer Series in Materials Science, Springer, Berlin, 1995.

3. H. Hirai and N. Toshima, in "Catalysis by Metal Complexes. Tailored Metal Catalysts," Y. Iwasawa, Ed., D. Reidel Publishing Company, Dordrecht, 1986.

4. H. Hirai, Y. Nakao, and N. Toshima, J. Macromol. Sci.-Chem., A13, 727 (1979).

5. M. Antonietti, E. Wenz, L. M. Bronstein, and M. Seregina, $A d v$. Mater., 7, 1000 (1995).

6. M. V. Seregina, L. M. Bronstein, O. A. Platonova, D. M. Chernyshov, P. M. Valetsky, E. Wenz, and M. Antonietti, Chem. Mater., 9, 923 (1997).
7. S. Klingelhöfer, W. Heitz, A. Greiner, S. Oestreich, S. Förster, and M. Antonietti, J. Am. Chem. Soc., 119, 10116 (1997).

8. O. A. Platonova, L. M. Bronstein, S. P. Solodovnikov, I. M. Yanovskaya, E. S. Obolonkova, P. M. Valetsky, E. Wenz, and M. Antonietti, Coll. Polym. Sci., 275, 426 (1997).

9. J. Yue and R. E. Cohen, Supramol. Sci., 1, 117 (1994).

10. R. T. Clay and R. E. Cohen, Supramol. Sci., 2, 183 (1995).

11. R. T. Clay and R. E. Cohen, Supramol. Sci., 4, 340 (1996).

12. R. T. Clay and R. E. Cohen, Supramol. Sci., 4, 113 (1997).

13. J. P. Spatz, A. Roescher, and M. Möller, Adv. Mater., 8, 337 (1996).

14. S. T. Selvan, J. P. Spatz, H.-A. Klok, and M. Möller, Adv. Mater. 10, 132 (1998)

15. A. Henglein, Ber. Bunsengesell. Phys. Chem., 101, 1562 (1997).

16. A. Henglein, Chem. Rev., 89, 1861 (1989).

17. A. D. Pomogailo, Platinum Metals Rev., 38, 60 (1994).

18. A. B. R. Mayer and J. E. Mark, J. Macromol. Sci., Pure Appl. Chem., A34, 2151 (1997).

19. A. B. R. Mayer and J. E. Mark, J. Polym. Sci., B: Polym. Phys., 35, 1207 (1997).

20. A. B. R. Mayer and J. E. Mark, J. Polym. Sci., A: Polym. Chem., 35, 3151 (1997).

21. A. B. R. Mayer, J. E. Mark, and R. E. Morris, Polym. J., 30, 197 (1998).

22. A. Henglein, P. Mulvaney, A. Holzwarth, T. E. Sosebee, and A. Fojtik, Ber. Bunsengesell. Phys. Chem., 96, 754 (1992).

23. P. C. Lee and D. Meisel, J. Catal., 70, 160 (1981).

24. A. Henglein, J. Phys. Chem., 83, 2209 (1979).

25. A. Henglein, J. Phys. Chem., 83, 2858 (1979).

26. H. Nakatskji, H. Nakai, K. Ikeda, and Y. Yamamoto, Surf. Sci., 384, 315 (1997).

27. H. Nakatskji, Z. M. Hu, H. Nakai, and K. Ikeda, Surf. Sci., 387, 328 (1997).

28. D. H. Napper, in "Polymeric Stabilization of Colloidal Dispersions," Academic Press, London, 1983.

29. "Gmelin Handbuch der Anorganischen Chemie, Silber, Teil B6," 8th ed, R. Kaim and B. Heibel, Ed., Springer, Berlin, 1975.

30. Z.-Y. Huang, G. Mills, and B. Hajek, J. Phys. Chem., 97, 11542 (1993).

31. C. Petit, P. Lixon, and M.-P. Pileni, J. Phys. Chem., 97, 12974 (1993).

32. "Gmelin Handbuch der Anorganischen Chemie, Silber, Teil B1," 8th ed, R. Kaim, Ed., Verlag Chemie, Weinheim, 1971.

33. A. Henglein, Chem. Mater., 10, 444 (1998).

34. A. B. R. Mayer, R. W. Johnston, S. H. Hausner, and J. E. Mark, submitted to J. Macromol. Sci., Part A-Pure Appl. Chem.

35. A. L. Rogach, G. P. Shevchenko, Z. M. Afanas'eva, and V. V. Sviridov, J. Phys. Chem. B, 101, 8129 (1997).

36. K,-P. Charlé, W. Schulze, and B. Winter, Z. Phys. D.-Atoms, Molecules, and Clusters, 12, 471 (1989).

37. A. Henglein, P. Mulvaney, T. Linnert, and A. Holzwarth, J. Phys. Chem., 96, 2411 (1992).

38. S. M. Heard, F. Grieser, and C. G. Barraclough, J. Coll. Interf. Sci., 93, 545 (1983).

39. A. Henglein, J. Phys. Chem., 97, 5457 (1993).

40. J. A. Creighton and D. G. Eadon, J. Chem. Soc., Faraday Trans., 87, 3881 (1991).

41. A. Henglein and R. Tausch-Treml, J. Coll. Interf. Sci., 80, 84 (1981).

42. J. Nedeljkovic and R. C. Patel, Appl. Phys. Lett., 58, 2461 (1991). 